\title{
A minimal nonfinitely based semigroup whose variety is polynomially recognizable
}

\author{
M. V. Volkov, S. V. Goldberg \\ Ural State University \\ email: \{Mikhail.Volkov,Svetlana.Goldberg\}@usu.ru \\ S. I. Kublanovsky \\ TPO "Severny Ochag" \\ email: stas@norths.spb.su
}

\begin{abstract}
We exhibit a 6-element semigroup that has no finite identity basis but nevertheless generates a variety whose finite membership problem admits a polynomial algorithm.
\end{abstract}

Keywords: semigroup, identity, variety, pseudovariety, finite basis property, membership problem, polynomial algorithm

\section{Motivation and overview}

Developments in the theory of computational complexity have shed new light on algorithmic aspects of algebra. It has turned out that many basic algorithmic questions whose decidability is well known and/or obvious give rise to fascinating and sometimes very hard problems if one looks for the computational complexity of corresponding algorithm 1 . A good example is provided by the problem VAR-MEMB studied in this paper: given two finite algebras $A$ and $B$ of the same similarity type, decide whether or not the algebra $A$ belongs to the variety generated by the algebra $B$. Clearly, the problem VAR-MemB is of importance for universal algebra in which equational classification of algebras is known to play a central role. At the same time, the problem is of interest in computer science and, in particular, in formal specification theory (cf. 1, Section 1]) and in formal language theory (see discussion below). The fact that the problem VAR-MEMB is decidable easily follows from Tarski's HSPtheorem and has already been mentioned in Kalicki's pioneering paper [9]. The question about computational complexity of this problem has been explicitly posed much later, namely, in Kharlampovich and Sapir's well-known survey, see [10, Problem 2.5]. A systematic study of this question has then started and

\footnotetext{
${ }^{1}$ In this paper complexity is understood in the sense of the monographs [5, 26]; the reader can find there the definitions of the complexity classes P, NP, EXPSPACE, and 2-EXPTIME that are mentioned below.
} 
brought interesting and rather unexpected results. Bergman and Slutzki [1] extracted an upper bound from an analysis of Kalicki's proof: the problem VAR-MEMB belongs to the class 2-EXPTIME of problems solvable in double exponential time. For some time it appeared that this bound was very loose but then Szekely [32] showed that the problem is NP-hard, and Kozik [13, 14] proved that it is even EXPSPACE-hard. Finally, Kozik [15] has shown that the problem VAR-MEMB is 2-EXPTIME-complete, thus confirming that the bound by Bergman and Slutzki in general is tight. Thus, the problem VAR-MEMB has turned out to be one of the hardest algorithmic problems of universal algebra.

The problem VAR-MEMB is of a special interest for semigroups in the view of the well-known Eilenberg correspondence [4] between varieties of regular language and pseudovarieties of semigroup\$2. By this correspondence, a regular language belongs to some language variety $\mathbf{L}$ if and only if the syntactic semigroup of the language belongs to the pseudovariety of semigroups corresponding to $\mathbf{L}$. Therefore, estimating complexity of the semigroup version of VAR-MEMB, one can deduce conclusions about computational complexity of some important problems in formal language theory. At the present moment, the precise complexity of the problem VAR-MEMB for semigroups has not yet been determined but it is known that the problem is NP-hard (Jackson and McKenzie [6]). In what follows, we concentrate on the problem VAR-MEMB for semigroups.

A reasonable strategy for analyzing VAR-MEMB in detail consists in fixing the semigroup $B$ as a parameter so that the role of an input is played by the semigroup $A$ only. This approach splits VAR-MEMB into a series of problems $\operatorname{VAR}-\operatorname{MemB}(B)$ that are parameterized by finite semigroups and leads to the question of classifying finite semigroups with respect to computational complexity of the membership problem for the varieties these semigroups generate. Let us proceed with precise definitions.

Let $B$ be an arbitrary but fixed finite semigroup and let var $B$ be the variety generated by $B$. The problem $\operatorname{VAR}-\operatorname{MemB}(B)$ is a combinatorial decision problem whose instance is an arbitrary finite semigroup $A$, and the answer to the instance $A$ is "YES" or "NO" depending on whether or not $A$ belongs to the variety $\operatorname{var} B$. If there exist a deterministic Turing machine and a polynomial $p(x)$ with integer coefficients, both depending on the semigroup $B$ only, such that for every finite semigroup $A$, the machine decides in time at most $p(|A|)$ whether or not $A$ belongs to the variety var $B$, then we say that $B$ is a semigroup with polynomially recognizable variety. Similarly, if there is no such polynomial, but there exists a real constant $\alpha>1$ such that for every finite semigroup $A$, the machine decides the same question in time at most $\alpha^{|A|}$, then we say that $B$ is a semigroup with exponentially recognizable variety, etc. The classification question mentioned in the previous paragraph is essentially the question of an efficient characterization of finite semigroups with polynomially (exponentially etc.) recognizable varieties. We notice that Jackson and McKenzie [6] have exhibited a 56-element semigroup $J M$ for which the problem $\operatorname{VAR-MemB}(J M)$

\footnotetext{
${ }^{2}$ Recall that a semigroup pseudovariety is a class of finite semigroups closed under taking subsemigroups and homomorphic images and under forming finitary direct products. In particular, the class $\mathcal{V}_{\text {fin }}$ of all finite semigroups in a given variety $\mathcal{V}$ is a pseudovariety.
} 
is NP-hard. This means that under the standard assumption $\mathrm{P} \neq \mathrm{NP}$, the semigroup $J M$ is not a semigroup with polynomially recognizable variety.

Semigroup with polynomially recognizable varieties could be alternatively called semigroups with easily verifiable identities. Indeed, by the definition the variety $\operatorname{var} B$ consists of all semigroups satisfying every identity holding in $B$, whence testing membership of a given semigroup $A$ in the variety $\operatorname{var} B$ is nothing but testing whether $A$ satisfies every identity of the semigroup $B$. This observation immediately implies a simple but important conclusion:

Lemma 1. If all identities holding in a semigroup $B$ follow from a finite set $\Sigma$ of such identities, then $B$ generates a polynomially recognizable variety.

Proof. Under the premise of the lemma, in order to check whether or not a given finite semigroup $A$ belongs to the variety $\operatorname{var} B$, it suffices to check whether or not $A$ satisfies all identities in $\Sigma$. To check that an identity $u=v$ in $\Sigma$ holds in $A$, provided that $u$ and $v$ together depend on $m$ variables, one can simply substitute for the variables all possible $m$-tuples of elements of $A$ and then check whether or not all substitutions yield equal values to the words $u$ and $v$. The number of $m$-tuples subject to the evaluation is $|A|^{m}$ while time needed to calculating the values of the words $u$ and $v$ depends only on the length of these words and not on the size of the semigroup $A$. Hence the total time consumed by this algorithm is bounded by a polynomial of degree $m$ in $|A|$. Since the number of identities in $\Sigma$ also does not depend on the size of $A$, we see that the inclusion $A \in \operatorname{var} B$ can be verified in polynomial in $|A|$ time.

A semigroup that satisfies the premise of Lemma 1 is said to be finitely based. The question which finite semigroups are finitely based and which are not plays a central role in the theory of semigroup varieties for more than 40 year, see [38] for a survey of the corresponding area. Lemma 1 links this question and the problem of characterizing finite semigroups with polynomially recognizable varieties.

It is easy to see that in general a polynomially recognizable variety need not be finitely based. Here the variety $\mathcal{B}_{4} \mathcal{B}_{2}$ of all extensions of groups of exponent 4 by groups of exponent 2 studied by Kleiman [11] can serve as a simple example. (Since this class consists of periodic groups, it also forms a semigroup variety.) Indeed, it is shown in [11] that the variety $\mathcal{B}_{4} \mathcal{B}_{2}$ is nonfinitely based. On the other hand, if $A$ is a finite semigroup, then in order to test the membership of $A$ in $\mathcal{B}_{4} \mathcal{B}_{2}$, it suffices to test whether or not $A$ is a group, and if this is the case, to check whether or not the normal subgroup generated by all squares in $A$ has exponent 4 . Clearly, both these checks can be performed in polynomial (in fact, cubic) in $|A|$ time.

The situation changes essentially if one considers a variety generated by a finite semigroup. Here one cannot find a similar example among varieties consisting only of groups because by a classic result by Oates and Powell [24] every finite group is finitely based. In [10, Theorem 3.53] the authors describe a certain semigroup variety $\mathcal{S}$ and claim that $\mathcal{S}$ is polynomially recognizable and that one can deduce from Sapir's result [29] that $\mathcal{S}$ is nonfinitely based and is 
generated by a finite semigroup. However, an algorithm for testing membership of a finite semigroup in the variety $\mathcal{S}$ is described in neither [10] nor subsequent publications; no finite semigroup generating $\mathcal{S}$ is explicitly exhibited. Moreover, the reference to [29] does not appear to be fully legitimate because it is clear from the description of the variety $\mathcal{S}$ in [10, Theorem 3.53] that all groups in $\mathcal{S}$ have exponent 4 while semigroup varieties analyzed in [29] contain only groups of odd exponent. We do not doubt the validity of the claim announced in [10. Theorem 3.53], but we believe nevertheless that in order to clarify the relationship between the properties of a finite semigroup "to be finitely based" and "to generate a polynomially recognizable variety", it is worthwhile to provide a more transparent example with complete justification. This is the goal of the present paper.

We exhibit a 6-element semigroup $A C_{2}$ that is nonfinitely based and at the same time generates a polynomially recognizable variety. We explicitly write down an infinite identity basis for $A C_{2}$ and describe in detail a polynomial algorithm for testing membership of an arbitrary finite semigroup in the variety $\operatorname{var} A C_{2}$.

We notice that our example has the minimum possible number of elements because it is well known that every semigroup with five or fewer elements is finitely based [34,35]. Surprisingly, it seems that the semigroup $A C_{2}$ has not yet appeared in the literature. The reader may be aware of the other 6-element nonfinitely based semigroup, the so-called Brandt monoid $B_{2}^{1}$ formed by the following $2 \times 2$-matrices

$$
\left(\begin{array}{ll}
0 & 0 \\
0 & 0
\end{array}\right), \quad\left(\begin{array}{ll}
1 & 0 \\
0 & 1
\end{array}\right), \quad\left(\begin{array}{ll}
1 & 0 \\
0 & 0
\end{array}\right), \quad\left(\begin{array}{ll}
0 & 1 \\
0 & 0
\end{array}\right), \quad\left(\begin{array}{ll}
0 & 0 \\
1 & 0
\end{array}\right),\left(\begin{array}{ll}
0 & 0 \\
0 & 1
\end{array}\right)
$$

under usual matrix multiplication. Since the pioneering paper by Perkins [27], the Brandt monoid appears over and over again in publications on the theory of semigroup varieties for more than 40 years. It is known that $B_{2}^{1}$ has many remarkable properties (including those related to computational complexity, see [12,30]) but the question about the complexity of the problem VAR$\operatorname{MemB}\left(B_{2}^{1}\right)$ still remains open (and is very intriguing in our opinion). Therefore at the moment one cannot use the Brandt monoid as the example we are looking for.

A further interesting property of the semigroup $A C_{2}$ is that var $A C_{2}$ is a limit variety, that is, a minimal (under class inclusion) nonfinitely based variety, see [18. Thus, our example is minimal not only with respect to the number of elements but also with respect to the natural ordering of varieties.

The paper is structured as follows. In Section 2 we construct the semigroup $A C_{2}$, establish its identity basis, and give a structural characterization of semigroups in the variety var $A C_{2}$. In Section 3 we show how to use this characterization in order to check, given a finite semigroup $S$, whether or not $S \in \operatorname{var} A C_{2}$ in time $O\left(|S|^{3}\right)$.

We assume the reader's acquaintance with rudiments of semigroup theory up to the first three chapters of the monograph [3] as well as with some basics of the theory of varieties, see [2, Chapter II]. For the reader's convenience we 
recall here the notion of a Rees matrix semigroup which is important for the present paper.

Let $G$ be a group, 0 be a symbol not in $G$. Further, let $I, \Lambda$ be non-empty sets and $P=\left(p_{\lambda, i}\right)$ be a $\Lambda \times I$-matrix over $G \cup\{0\}$. The Rees matrix semigroup $M^{0}(G ; I, \Lambda ; P)$ over the group $G$ with the sandwich-matrix $P$ is the semigroup on the set $(I \times G \times \Lambda) \cup\{0\}$ under multiplication

$$
\begin{aligned}
& x \cdot 0=0 \cdot x=0 \text { for all } x \in(I \times G \times \Lambda) \cup\{0\}, \\
& (i, g, \lambda) \cdot(j, h, \mu)= \begin{cases}0 & \text { if } p_{\lambda, j}=0, \\
\left(i, g p_{\lambda, j} h, \mu\right) & \text { if } p_{\lambda, j} \neq 0 .\end{cases}
\end{aligned}
$$

\section{The semigroup $A C_{2}$ and its identity basis}

Let $A_{2}$ denote the 5-element idempotent-generated 0 -simple semigroup. It can be defined in the class of semigroups with zero by the following presentation:

$$
A_{2}=\left\langle a, b \mid a^{2}=a b a=a, b a b=b, b^{2}=0\right\rangle=\{a, b, a b, b a, 0\} .
$$

The semigroup $A_{2}$ can be also thought of as the semigroup formed by the following $2 \times 2$-matrices (over an arbitrary field)

$$
\left(\begin{array}{ll}
0 & 0 \\
0 & 0
\end{array}\right),\left(\begin{array}{ll}
1 & 0 \\
0 & 0
\end{array}\right),\left(\begin{array}{ll}
0 & 1 \\
0 & 0
\end{array}\right),\left(\begin{array}{ll}
1 & 0 \\
1 & 0
\end{array}\right),\left(\begin{array}{ll}
0 & 1 \\
0 & 1
\end{array}\right)
$$

under the usual multiplication of matrices or as the Rees matrix semigroup over the trivial group $E=\{1\}$ with the sandwich-matrix $\left(\begin{array}{ll}1 & 1 \\ 0 & 1\end{array}\right)$.

The semigroup $A C_{2}$ is obtained by adding a new element $c$ to the semigroup $A_{2}$. The multiplication in $A C_{2}$ extends the multiplication in $A_{2}$ and the products involving the added element $c$ are defined as follows:

$$
c^{2}=0 \text { and } x c=c x=c \text { for all } x \in A_{2} .
$$

(In order to avoid any confusion, we stress that the element 0 is no longer a zero in $A C_{2}$ since $0 c=c 0=c$.) The fact that the multiplication defined this way is associative can be straightforwardly verified but can be also obtained without calculations if one observes that the groupoid $A C_{2}$ is isomorphic to a subsemigroup of the direct product of the semigroup $A_{2}$ with the cyclic group $C_{2}=\left\langle c \mid c^{2}=1\right\rangle=\{c, 1\}$, namely, to the subsemigroup consisting of all the pairs of the form $(x, 1)$, where $x \in A_{2}$, and the pair $(0, c)$.

By the construction, $A_{2}$ is a subsemigroup in $A C_{2}$; on the other hand, the elements 0 and $c$ form in $A C_{2}$ a subgroup isomorphic to the group $C_{2}$. (Thus, $A C_{2}$ is obtained via amalgamating $A_{2}$ and $C_{2}$ such that the zero of the semigroup $A_{2}$ is identified with the identity element of the group $C_{2}$.) Since $A_{2}, C_{2} \in \operatorname{var} A C_{2}$, we have $A_{2} \times C_{2} \in \operatorname{var} A C_{2}$. Conversely, as mentioned above, the semigroup $A C_{2}$ embeds into the direct product $A_{2} \times C_{2}$ whence $A C_{2} \in \operatorname{var}\left(A_{2} \times C_{2}\right)$. We see that the semigroups $A C_{2}$ and $A_{2} \times C_{2}$ generate the same variety, in other words, they satisfy the same identities. It follows from the results of [37] (cf. Remark 2 in the discussion of the main theorem 
there) that for every group $G$ of finite exponent the direct product $A_{2} \times G$ is nonfinitely based. Hence we obtain the first property of the semigroup $A C_{2}$ we need.

Lemma 2. The semigroup $A C_{2}$ is nonfinitely based.

Remark 1. The short note [20] contains an announcement (with a proof sketch) of the following fact: the Rees matrix semigroup over the group $C_{2}$ with the sandwich-matrix $\left(\begin{array}{ll}1 & 1 \\ 0 & 1\end{array}\right)$ is nonfinitely based. It is not hard to show that this 9-element semigroup generates the same variety as the semigroups $A C_{2}$ and $A_{2} \times C_{2}$. Therefore Lemma 2 can also be deduced from the result of [20].

Now we describe the identities of the semigroup $A C_{2}$. For a word $w$, we denote $\operatorname{by} \operatorname{alph}(w)$ the set of variables that occur in $w$ and by $\left|w_{x}\right|$ the number of occurrences of the variable $x$ in $w$. Given a word $w$, we assign to it a directed graph $G(w)$ whose vertex set is $\operatorname{alph}(w)$ and whose edges correspond to factors of length 2 in $w$ as follows: $G(w)$ has a directed edge from $x$ to $y(x, y \in \operatorname{alph}(w))$ if and only if some occurrence of $x$ in $w$ immediately precedes some occurrence of $y$. We will distinguish two (not necessarily different) vertices in $G(w)$ : the

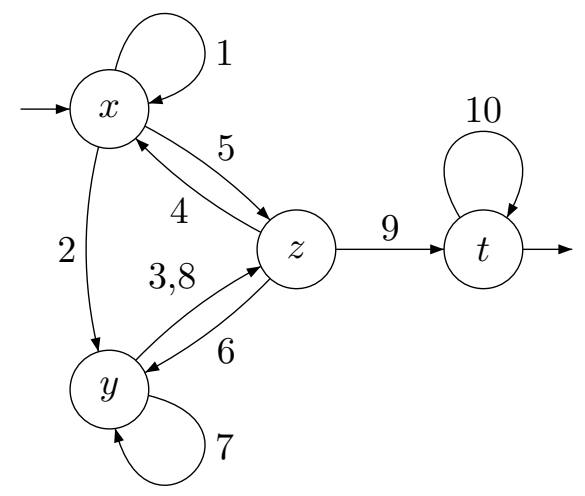

Figure 1: The graph of the word $w=x^{2} y z x z y^{2} z t^{2}$ and the corresponding walk

initial vertex, that is the first letter of $w$, and the final vertex, that is the last letter of $w$. Then the word $w$ defines a walk through the graph $G(w)$ that starts at the initial vertex, ends at the final vertex and traverses each edge of $G(w)$ (some of the edges can be traversed more than once).

Figure 1 shows the graph $G(w)$ for the word $w=x^{2} y z x z y^{2} z t^{2}$. The ingoing and the outgoing marks show respectively the initial and the final vertices of the graph. In Fig. 1 each edge of the graph is labelled by the number[s] corresponding to the occurrence[s] of the edge in the walk induced by the word $w$. We stress that, in contrast to the vertex names and the ingoing/outgoing marks, these labels are not considered as a part of the data making the graph $G(w)$. Therefore the graph does not determine the word $w$ : for instance, as the reader can easily check, the word $x y^{3} z y z x^{2} z y z t^{3}$ has exactly the same graph (but corresponds to a different walk through it, see Fig.21).

Observe that in terms of the graph $G(w)$, the number $\left|w_{x}\right|$ represents the number of times that the walk induced by the word $w$ visits the vertex $x$. 


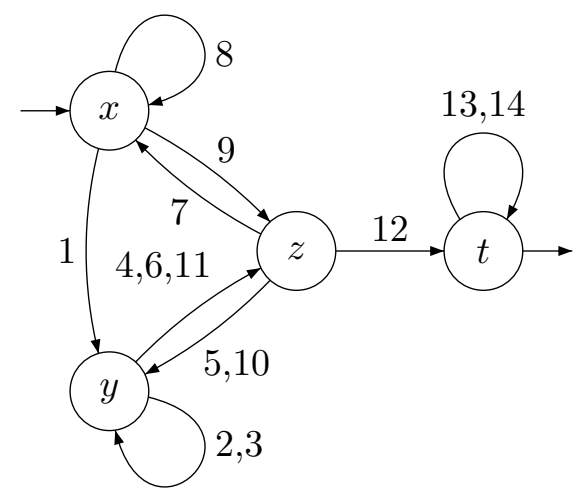

Figure 2: Another walk through the graph of Fig.1

Proposition 3. An identity $u=v$ holds true in the semigroup $A C_{2}$ if and only if the graphs $G(u)$ and $G(v)$ coincide and, for each variable $x$, the numbers $\left|u_{x}\right|$ and $\left|v_{x}\right|$ have the same parity.

Proof. We have mentioned above that the semigroups $A C_{2}$ and $A_{2} \times C_{2}$ satisfy the same identities. Clearly, an identity holds in the semigroup $A_{2} \times C_{2}$ if and only if it holds in each of the semigroups $A_{2}$ and $C_{2}$. It is known that an identity $u=v$ holds true in the semigroup $A_{2}$ if and only if the graphs $G(u)$ and $G(v)$ coincide, see [33] 3 . Further, it is known (and easy to verify) that an identity $u=v$ holds true in the group $C_{2}$ if and only if the numbers $\left|u_{x}\right|$ and $\left|v_{x}\right|$ have the same parity for each variable $x$.

Proposition 3 immediately implies

Corollary 4. The identities

$$
\begin{gathered}
x^{2}=x^{4} \\
x y x=(x y)^{3} x, \\
x y x z x=x z x y x \\
\left(x_{1}^{2} x_{2}^{2} \cdots x_{n}^{2}\right)^{2}=\left(x_{1}^{2} x_{2}^{2} \cdots x_{n}^{2}\right)^{3}, \quad n=2,3, \ldots,
\end{gathered}
$$

hold true in the semigroup $A C_{2}$.

Proof. It is easy to see that for each of the identities (11)-(4), the graph of its left hand side coincides with the graph of its right hand side (the graphs are shown in Fig.31) and each variable occurs on the left and on the right with the same parity.

We aim to clarify the structural meaning of the identities (1)-(4). We start with the series (4). Recall that a semigroup is said to be combinatorial if all of its subgroups are singletons.

\footnotetext{
${ }^{3}$ In the literature (see, for instance, 16 or 36 ) one sometimes refers to 19 as the source for this result even though the paper [19] does not deal with the semigroup $A_{2}$ at all. Apparently, this mistake originates from an erroneous reference in the survey paper [31.
} 


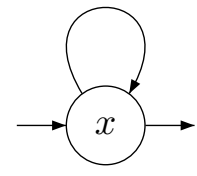

(11)
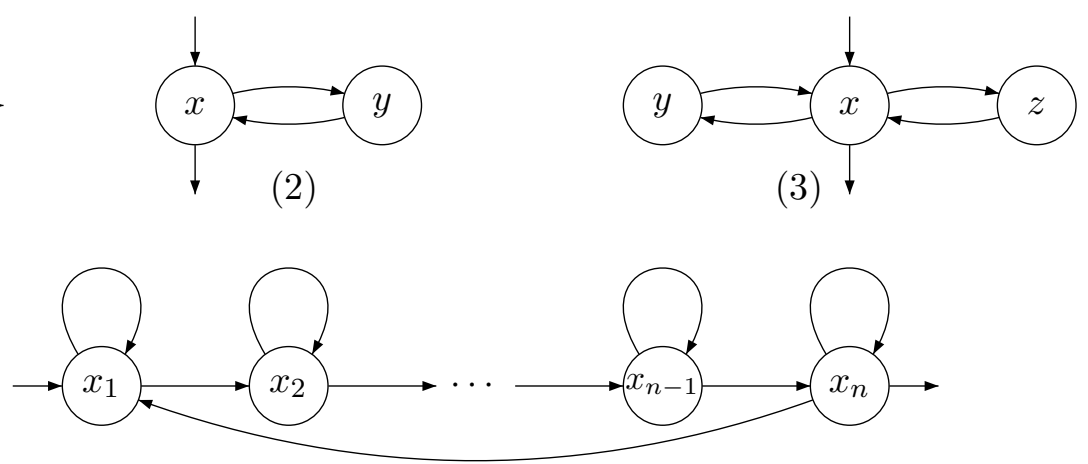

(41)

Figure 3: The graphs of the identities (10)-(4)

Proposition 5. If a semigroup $S$ satisfies the identities (4), then the subsemigroup generated by all idempotents of $S$ is combinatorial. If $S$ satisfies the identity (1), then the converse is true as well.

Proof. Since every idempotent can be represented as a square, products of the form $x_{1}^{2} \cdots x_{n}^{2}, n=2,3, \ldots$, represent all elements of the subsemigroup $T$ generated by all idempotents of $S$. If $S$ satisfies (4), then $T$ satisfies the identity

$$
x^{2}=x^{3}
$$

that cannot hold in a non-singleton group. Hence, the subsemigroup $T$ is combinatorial.

Conversely, let $S$ satisfies the identity (1). Then the subsemigroup $T$ also satisfies this identity but in a combinatorial semigroup (11) implies (5). In the presence of the identity (1), the square of each element of $S$ is an idempotent whence the values of all products of the form $x_{1}^{2} \cdots x_{n}^{2}, n=2,3, \ldots$, lie in $T$. Substituting these products for the variable in (5), we see that $S$ satisfies all identities from the series (4).

The variety generated by all completely 0 -simple semigroups whose subgroups have exponent $n$ is commonly denoted by $\mathcal{R} \mathcal{S}_{n}$. Clearly, the semigroup $A C_{2}$ belongs to the variety $\mathcal{R S}_{2}$. The next results reveals the role of the identities (1)-(3):

Proposition 6. The identities (10)-(3) form an identity basis of the variety $\mathcal{R S}_{2}$.

We do not prove Proposition [6 here because it is not used in the present paper. We notice that various identity bases for $\mathcal{R} \mathcal{S}_{n}$ have been provided in [7, [21,23] 4. Unfortunately, the proofs of the corresponding results in these papers cannot be considered as complete because they all essentially use a lemma from [21] whose proof in [21] is wrong. We shall discuss these nuances in the

\footnotetext{
${ }^{4}$ However, the identity basis for $\mathcal{R} \mathcal{S}_{2}$ specified in Proposition 6 is not a specialization of the bases for $\mathcal{R S}_{n}$ provided in [7,21,23].
} 
course of the proof of the next theorem that plays a key role in the present paper.

Theorem 1. The identities (11) -(4) form an identity basis for $A C_{2}$.

The proof follows a scheme suggested in [17]. We need a few auxiliary statements.

A word $w$ of length at least 2 is said to be connected if its graph $G(w)$ is strongly connected 5 . Let $\mathcal{V}$ be the variety defined by the identities (10)-(4).

Lemma 7. If $w$ is a connected word and $S$ is a semigroup in $\mathcal{V}$, then every value of $w$ in $S$ is a regular element in $S$.

Proof. We recall that an element $s \in S$ is said to be regular in $S$ if there exists an element $s^{\prime} \in S$ such that $s s^{\prime} s=s$. Therefore in order to prove the lemma it suffices to construct a word $w^{\prime}$ such that the variety $\mathcal{V}$ satisfies the identity $w=w w^{\prime} w$. If the word $w$ begins and ends with the same variable, then we can apply the identity (2) to it (or the identity (11) in the case when $w$ is the square of a variable) and we immediately get the necessary conclusion. We may therefore assume that $w$ begins with a variable $x$ and ends with a variable $y$ such that $x \neq y$.

Since the word $w$ is connected, each of the variables $x$ and $y$ occurs in $w$ more than once. We want to show that, applying the identities (2) and (3), one can transform $w$ into a word in which some occurrence of the variable $x$ appears after some occurrence of the variable $y$. For this, it is convenient to prove a slightly more general lemma.

Lemma 8. Let $w$ be a connected word, $x, y \in \operatorname{alph}(w)$ and

$$
w=w_{1} x w_{2} y w_{3}, \quad x \notin \operatorname{alph}\left(w_{2} y w_{3}\right) \text { and } y \notin \operatorname{alph}\left(w_{1} x w_{2}\right) .
$$

Applying the identities (2) and (3), one can transform $w$ into a word $w_{1} x w_{2}^{\prime} y w_{3}$ such that $x, y \in \operatorname{alph}\left(w_{2}^{\prime}\right)$ and some occurrence of the variable $x$ in $w_{2}^{\prime}$ appears after some occurrence of the variable $y$ in $w_{2}^{\prime}$

Proof. First of all, we observe that if some occurrences of the variables $x$ and $y$ happen between two occurrences of some variable $z$, then the desired

\footnotetext{
${ }^{5}$ This concept sometimes appears in the literature under different names. For instance, in 21] a word $w$ of length at least 2 is said to be covered by cycles each if each of its factors of length 2 occurs in a factor of $w$ that begins and ends with a common letter. In the language of the graph $G(w)$, this means that each directed edge $x \rightarrow y$ of $G(w)$ belongs to a directed cycle (namely, to the walk induced by a factor of $w$ that starts and ends with the same letter and contains $x y$ ). It is one of the basic facts of the theory of directed graphs (cf. [25], Theorem 8.1.5) that such a graph is strongly connected if and only if each of its directed edges belongs to a directed cycle. Thus, words covered by cycles in the sense of 21] are precisely connected words in our sense. Yet another name for an obviously equivalent concept has been introduced in 28, where a word $w$ of length at least 2 is said to be prime if it cannot be decomposed as $w=w^{\prime} w^{\prime \prime}$ with $\operatorname{alph}\left(w^{\prime}\right) \cap \operatorname{alph}\left(w^{\prime \prime}\right)=\varnothing$.
} 
transformation can be achieved by an application of the identity (2) to the factor bordered by these two occurrences of $z$ :

$$
\begin{aligned}
w & =\underbrace{w_{11} z w_{12}}_{w_{1}} x w_{2} y \underbrace{w_{31} z w_{32}}_{w_{3}}=w_{11}\left(z w_{12} x w_{2} y w_{31}\right)^{3} z w_{32} \\
& =\underbrace{w_{11} z w_{12}}_{w_{1}} x w_{2} y w_{31} z w_{12} x w_{2} \underline{\underline{y}} w_{31} z w_{12} \underline{\underline{x}} w_{2} y \underbrace{w_{31} z w_{32}}_{w_{3}} .
\end{aligned}
$$

(The "permuted" occurrences of the variables $x$ and $y$ are underlined twice.)

Now we induct on the length of the word $w_{2}$ in the decomposition (6), that is, on the distance between the right most occurrence of $x$ and the left most occurrence of $y$. If this distance is equal to 0 , then the word $w$ has $x y$ as a factor. Since $w$ is connected, this factor should appear between two occurrences of some variable $z$, and then the argument from the previous paragraph applies. This proves the induction basis.

Now suppose that in the decomposition (6) the word $w_{2}$ is not empty. In view of the first paragraph of the proof, we can assume that $\operatorname{alph}\left(w_{1} x\right) \cap$ $\operatorname{alph}\left(y w_{3}\right)=\varnothing$. Since $w$ is connected, the word $w_{2}$ must have common variables with each of the words $w_{1}$ and $w_{3}$. Consider two cases.

Case 1. Some variable $z \in \operatorname{alph}\left(w_{1}\right) \cap \operatorname{alph}\left(w_{2}\right)$ occurs in the word $w_{2}$ to the left of some variable $t \in \operatorname{alph}\left(w_{2}\right) \cap \operatorname{alph}\left(w_{3}\right)$.

In this case, the desired transformations are as follows:

$$
\begin{aligned}
& w=\underbrace{w_{11} z w_{12}}_{w_{1}} x \underbrace{w_{21} t w_{22} z w_{23}}_{w_{2}} y \underbrace{w_{31} t w_{32}}_{w_{3}} \\
& =w_{11}\left(z w_{12} x w_{21} t w_{22} z\right)^{3} w_{23} y w_{31} t w_{32} \quad \text { (by (2) ) } \\
& =w_{11} z w_{12} x w_{21} t w_{22} z w_{12} x w_{21} t w_{22} z w_{12} x w_{21} t w_{22} z w_{23} y w_{31} t w_{32} \\
& \left.=w_{11} z w_{12} x w_{21} t w_{22} z w_{12} x w_{21} t w_{22} z w_{12} x w_{21}\left(t w_{22} z w_{23} y w_{31}\right)^{3} t w_{32} \quad \text { (by (2) }\right) \\
& =w_{11} z w_{12} x w_{21} t w_{22} z w_{12} x w_{21} \underline{t w_{22} z w_{12} x w_{21} t w_{22} z w_{23} y w_{31} t} \times \\
& \times w_{22} z w_{23} y w_{31} t w_{22} z w_{23} y w_{31} t w_{32} \\
& =\underbrace{w_{11} z w_{12}}_{w_{1}} x w_{21} t w_{22} z w_{12} x w_{21} t w_{22} z w_{23} \underline{\underline{y}} \underline{\underline{w}} w_{31} t w_{22} z w_{12} \underline{\underline{x}} w_{21} t \times \\
& \times w_{22} z w_{23} y w_{31} t w_{22} z w_{23} y \underbrace{w_{31} t w_{32}}_{w_{3}}
\end{aligned}
$$

(The factor to which the identity (3) has been applied is underlined once while the "permuted" occurrences of the variables $x$ and $y$ are underlined twice.)

Case 2. Every variable from $\operatorname{alph}\left(w_{1}\right) \cap \operatorname{alph}\left(w_{2}\right)$ occurs in the word $w_{2}$ to the right of every variable from $\operatorname{alph}\left(w_{2}\right) \cap \operatorname{alph}\left(w_{3}\right)$.

We take some variables $z \in \operatorname{alph}\left(w_{1}\right) \cap \operatorname{alph}\left(w_{2}\right)$ and $t \in \operatorname{alph}\left(w_{2}\right) \cap \operatorname{alph}\left(w_{3}\right)$. Since both $z$ and $t$ occur in the word $w_{2}$ while both $x$ and $y$ do not, the distance between the right most occurrence of the variable $z$ and the left most occurrence of the variable $t$ is less than distance between the right most occurrence of the variable $x$ and the left most occurrence of the variable $y$. Thus, we can apply the induction assumption to the word $w$ and the variables $z, t$. This means that 
if we write the word $w$ as

$$
w=v_{1} z v_{2} t v_{3}, \quad z \notin \operatorname{alph}\left(v_{2} t v_{3}\right) \text { and } t \notin \operatorname{alph}\left(v_{1} z v_{2}\right),
$$

then applying the identities (2) and (3), we can transform $w$ into a word $v=$ $v_{1} z v_{2}^{\prime} t v_{3}$ such that $z, t \in \operatorname{alph}\left(v_{2}^{\prime}\right)$ and some occurrence of the variable $z$ in $v_{2}^{\prime}$ appears after some occurrence of the variable $t$ in $v_{2}^{\prime}$. However the word $v$ and the initial variables $x, y$ then satisfy the condition of Case 1 that is considered above.

Now we return to the proof of Lemma 7, Recall that we consider a connected word $w$ that begins with a variable $x$ and ends with a variable $y$ such that $x \neq y$. By Lemma 8 we may assume that some occurrence of the variable $x$ in $w$ appears after some occurrence of the variable $y$ in $w$. Hence

$$
\begin{aligned}
& w=x w_{1} y w_{2} x w_{3} y=\left(x w_{1} y w_{2}\right)^{3} x w_{3} y \\
& =x w_{1} y w_{2} x w_{1} y w_{2} x w_{1} y w_{2} x w_{3} y \\
& =x w_{1} y w_{2} x w_{1} y w_{2} x w_{1}\left(y w_{2} x w_{3}\right)^{3} y
\end{aligned}
$$

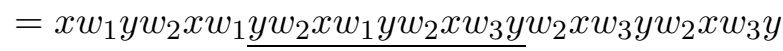

$$
\begin{aligned}
& \left.=x w_{1} \underline{y w_{2} x w_{1} y w_{2} x w_{3} y} w_{2} x w_{1} y w_{2} x w_{3} y w_{2} x w_{3} y \quad \text { (by (3) }\right)
\end{aligned}
$$

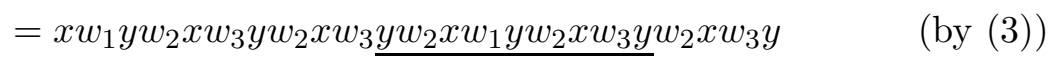

$$
\begin{aligned}
& =\underbrace{x w_{1} y w_{2} x w_{3} y}_{w} \underbrace{w_{2} x w_{1} y w_{2} x w_{3} y w_{2}}_{w^{\prime}} \underbrace{x w_{1} y w_{2} x w_{3} y}_{w} \quad \text { (by (3) ). }
\end{aligned}
$$

(The factors to which the identity (3) has been applied are underlined.) Thus, we have deduced an identity of the form $w=w w^{\prime} w$ from (11)-(3), as required.

Remark 2. Lemma 7 is a partial case of a similar result claimed by Mashevitsky in [21, Lemma 6], see also [22, Lemma 7]. As we have already mentioned, this result has been used (with reference to [21]) in several important papers, in particular, 7] and [23]. However, its proof in [21] contains a fatal flaw and so does the English translation of the proof published in 22. Namely, in 21] Lemma 6 is deduced from Lemma 5 which claims that every word $u$ covered by cycles can be transformed modulo certain identities into a word of the form $z_{1} u_{1} z_{1} \cdots z_{k} u_{k} z_{k}$ where $z_{1}, \ldots, z_{k}$ are variables and $z_{i+1} \in \operatorname{alph}\left(u_{i}\right)$ for all $i=1, \ldots, k-1$ provided that $k>1$. In order to justify the latter claim, Mashevitsky inducts on $|\operatorname{alph}(u)|$ but in the course of the proof he illegitimately applies the induction assumption to a factor that in general is not covered by its cycles. The word $x y x z y$ can be used as a concrete counter example showing that the argument from [21 does not work: here the induction assumption should have been applied to the factor $z y$ which is certainly not covered by its cycles.

We observe that our proof of Lemma 7 invokes only the identities (1)-(3). Some modification of our argument applies also to the identities considered in [21] and allows one to prove Lemma 6 of [21]. Thus, results of [7] and [23] 
that rely on the lemma are correct. Moreover, the third author of the present paper has recently proved that already the identities (11) and (2) suffice to ensure that the value of every connected word is regular; an analogous generalization also holds in the situation considered in [21].

A semigroup $S$ is called $E$-separable if for every pair $p, q$ of distinct elements in $S$, there exist idempotents $e, f \in S$ such that $p e \neq q e$ and $f p \neq f q$.

Lemma 9. The semigroup $A C_{2}$ is E-separable.

Proof. This amounts to filling out the following table where for each pair $p, q$ of distinct elements in the semigroup $A C_{2}$, we exhibit some idempotents $e$ and $f$ that separate $p$ and $q$ respectively on the right and on the left.

\begin{tabular}{|c||c|c|c|c|c|c|c|c|}
\hline$p$ & $c$ & 0 & $a$ & $a$ & $a$ & $a b$ & $a b$ & $b a$ \\
\hline$q$ & $x \in A_{2}$ & $y \in A_{2} \backslash\{0\}$ & $b$ & $a b$ & $b a$ & $b$ & $b a$ & $b$ \\
\hline \hline$e$ & 0 & $a$ & $a$ & $b a$ & $a$ & $a$ & $a$ & $b a$ \\
\hline$p e$ & $c$ & 0 & $a$ & $a$ & $a$ & $a$ & $a$ & $b a$ \\
\hline$q e$ & 0 & $a$ or $b a$ & $b a$ & 0 & $b a$ & $b a$ & $b a$ & 0 \\
\hline \hline$f$ & 0 & $a$ & $a$ & $a$ & $a b$ & $a b$ & $a$ & $b a$ \\
\hline$f p$ & $c$ & 0 & $a$ & $a$ & $a$ & $a b$ & $a b$ & $b a$ \\
\hline$f q$ & 0 & $a$ or $a b$ & $a b$ & $a b$ & 0 & 0 & $a$ & $b$ \\
\hline
\end{tabular}

The next result that we need is the union of the first part of Proposition 3.2 in [17] with the dual statement. By $A_{0}$ we denote the subsemigroup $A_{2} \backslash\{a\}=$ $\{b, a b, b a, 0\}$ of the semigroup $A_{2}$.

Lemma 10. Let $S$ be an E-separable semigroup and $A_{0} \in \operatorname{var} S$. Suppose that $S$ satisfies an identity $u=v$ such that the word $u$ can be represented as $u_{1} u_{2}$ with $\operatorname{alph}\left(u_{1}\right) \cap \operatorname{alph}\left(u_{2}\right)=\varnothing$. Then the word $v$ can be represented as $v_{1} v_{2}$ such that $\operatorname{alph}\left(v_{1}\right)=\operatorname{alph}\left(u_{1}\right), \operatorname{alph}\left(v_{2}\right)=\operatorname{alph}\left(u_{2}\right)$ and the semigroup $S$ satisfies the identities $u_{1}=v_{1}$ and $u_{2}=v_{2}$.

The next lemma is borrowed from [7], see Lemma 3.2 there.

Lemma 11. If for some $n \geq 1$ a semigroup $S$ satisfies the identities

$$
x^{2}=x^{n+2}, x y x=(x y)^{n+1} x, x y x(z x)^{n}=x(z x)^{n} y x,
$$

then for every pair of distinct regular elements $p, q \in S$ there exist a completely 0 -simple semigroup $K$ and a surjective homomorphism $\chi: S \rightarrow K$ such that $p \chi \neq q \chi$.

The last ingredient of our proof is a well-known result by Houghton 8 , Theorem 5.1] formulated in a convenient for us way.

Lemma 12. If the idempotents of a completely 0-simple semigroup $S$ generate a combinatorial subsemigroup, then $S$ can be presented as the Rees matrix semigroup $M^{0}(G ; I, \Lambda ; P)$ over a group $G$ such that every entry of the sandwich-matrix $P$ is equal to either zero or the identity element of $G$. 
Proof of Theorem 1. Recall that we have denoted by $\mathcal{V}$ the variety defined by the identities (1) -(4). By Corollary 4 we have the inclusion var $A C_{2} \subseteq \mathcal{V}$. Arguing by contradiction, assume that this inclusion is strict. Then there exists an identity that holds in the semigroup $A C_{2}$ but fails in the variety $\mathcal{V}$. Among all such identities, we chose an identity $u=v$ with the minimum possible number of variables in the word $u$. We aim to show that the words $u$ and $v$ must be connected.

Assume for the moment that, say, $u$ is not connected. This means that it can be decomposed as $u=u_{1} u_{2}$ with $\operatorname{alph}\left(u_{1}\right) \cap \operatorname{alph}\left(u_{2}\right)=\varnothing$. By Lemma 9 the semigroup $A C_{2}$ is $E$-separable, and since $A C_{2}$ obviously contains the semigroup $A_{0}$ as a subsemigroup, we see that Lemma 10 applies to $A C_{2}$. By this lemma we have $v=v_{1} v_{2}$ where $\operatorname{alph}\left(v_{1}\right)=\operatorname{alph}\left(u_{1}\right), \operatorname{alph}\left(v_{2}\right)=\operatorname{alph}\left(u_{2}\right)$ and both $u_{1}=v_{1}$ and $u_{2}=v_{2}$ hold in the semigroup $A C_{2}$. Since $\left|\operatorname{alph}\left(u_{1}\right)\right|,\left|\operatorname{alph}\left(u_{2}\right)\right|<$ $|\operatorname{alph}(u)|$, the choice of the identity $u=v$ ensures that the identities $u_{1}=v_{1}$ and $u_{2}=v_{2}$ hold in the variety $\mathcal{V}$. Clearly, the identity $u=v$ is a consequence of these two identities whence it also must hold in $\mathcal{V}$, a contradiction. Analogously, one checks that the word $v$ must be connected.

Now let $S$ be a semigroup in $\mathcal{V}$ such that the words $u$ and $v$ take distinct values $p$ and $q$ under some interpretation of variables. By Lemma 7 these values are regular elements. If we compare the identities that define the variety $\mathcal{V}$ with the three identities (7) from the premise of Lemma 11, we see that for $n=2$ the first two of the three identities coincide with the identities (1) and (2) respectively while the third one readily follows from the identity (3). Thus, Lemma 11 applies to the semigroup $S$ and its regular elements $p$ and $q$. Therefore there exist a completely 0 -simple semigroup $K$ and a surjective homomorphism $\chi: S \rightarrow K$ such that $p \chi \neq q \chi$. Observe that the elements $p \chi$ and $q \chi$ are also values of the words $u$ and $v$ under some interpretation of variables whence the identity $u=v$ fails in the semigroup $K$. On the other hand, the semigroup $K$ belongs to the variety $\mathcal{V}$ because it is a homomorphic image of the semigroup $S \in \mathcal{V}$. This means that we can use $K$ instead of $S$; in other words, we may (and will) assume that the semigroup $S$ from the "gap" between the varieties var $A C_{2}$ and $\mathcal{V}$ is completely 0 -simple.

By Proposition 5 the idempotents of $S$ generate a combinatorial subsemigroup, but then Lemma 12 implies that $S$ can be presented as the Rees matrix semigroup $M^{0}(G ; I, \Lambda ; P)$ over a group $G$ such that every entry of the sandwichmatrix $P$ is equal to either zero or the identity element of $G$. Let $T$ be the Rees matrix semigroup $M^{0}(E ; I, \Lambda ; P)$ over the trivial group $E=\{1\}$ with the same sandwich-matrix $P$. It is known (see, e.g., [17, Proposition 1.2]) that every completely 0 -simple semigroup over the trivial group belongs to the variety generated by the semigroup $A_{2}$; in particular, $T \in \operatorname{var} A_{2}$. Further, the group $G$ is isomorphic to a maximal subgroup in $S$ whence $G \in \mathcal{V}$. Therefore $G$ satisfies the identity (11) and hence $G$ is a group of exponent 2. It is well-known that every group of exponent 2 belongs to the variety generated by the group $C_{2}$; in particular, $G \in \operatorname{var} C_{2}$.

It is easy to verify that the mapping $T \times G \rightarrow S$ that sends the pair $((i, 1, \lambda), g) \in T \times G$ to the element $(i, g, \lambda) \in S$ is a surjective homomorphism. 
Since $T \in \operatorname{var} A_{2}$ and $G \in \operatorname{var} C_{2}$, we have

$$
T \times G \in \operatorname{var}\left(A_{2} \times C_{2}\right)=\operatorname{var} A C_{2},
$$

whence $S \in \operatorname{var} A C_{2}$. This contradicts the choice of the semigroup $S$. The theorem is proved.

\section{A polynomial algorithm for $\operatorname{Var}-\operatorname{Memb}\left(A C_{2}\right)$}

Given a semigroup $S$ with $|S|=n$, we want to test whether or not $S$ belongs to the variety $\operatorname{var} A C_{2}$. For this, by Theorem 1, it is necessary and sufficient to test whether or not $S$ satisfies the identities (1)-(4). Testing the identities (1) -(3) requires $O\left(n^{3}\right)$ time, see the argument in the proof of Lemma 1. No straightforward test for the infinite identity series (4) is possible but here we can use the structural equivalent from Proposition 5: it is necessary and sufficient to test whether or not the subsemigroup of the semigroup $S$ generated by all idempotents of $S$ is combinatorial. We will show that this can also be tested in $O\left(n^{3}\right)$ time.

Calculating squares of all elements of the semigroup $S$, we can find the set of all idempotents in $S$ in $O(n)$ time. Let $T_{1}$ be this set and define inductively $T_{i+1}=T_{i} T_{1}$. It is clear that constructing each set $T_{i+1}$ requires at most $n^{2}$ steps. Further, it is easy to see that $T_{i} \subseteq T_{i+1}$ and that if $T_{k}=T_{k+1}$ for some $k$, then $T_{k}=T_{k+\ell}$ for all $\ell$ whence $T_{k}$ is a subsemigroup in $S$. By the construction, every element in $T_{k}$ is a product of idempotents, and therefore, $T_{k}$ coincides with the subsemigroup of the semigroup $S$ generated by all idempotents of $S$. Since no strictly increasing chain of subsets of $S$ can contain more than $n$ subsets, we have $k \leq n$, and the subsemigroup $T_{k}$ will be constructed this way in $O\left(n^{3}\right)$ time. Now it remains to test whether or not $T_{k}$ is combinatorial and for this it is necessary and sufficient to test whether or not $T_{k}$ satisfies the identity (5), see the proof of Proposition 5. This last check can be done in $O(n)$ time.

Thus, we have proved the main result of the present paper:

Theorem 2. The 6-element semigroup $A C_{2}$ has no finite identity basis but, given a finite semigroup $S$, one can test the membership of $S$ in the variety var $A C_{2}$ in $O\left(|S|^{3}\right)$ time.

Acknowledgement. The first and the second authors acknowledge support from the Federal Education Agency of Russia, project 2.1.1/3537, and from the Russian Foundation for Basic Research, grants 09-01-12142 and 10-01-00524.

\section{References}

[1] Bergman C., Slutzki G. Complexity of some problems concerning varieties and quasi-varieties of algebras // SIAM J. Comput. - 2000. - Vol. 30, no. 2. - P. 359382.

[2] Burris S., Sankappanavar H. P. A Course in Universal Algebra. - Berlin: SpringerVerlag, 1981. 
[3] Clifford A. H., Preston G. B. The Algebraic Theory of Semigroups. Vol. 1. - Providence: Amer. Math. Soc., 1961.

[4] Eilenberg S. Automata, Languages and Machines. Vol. B. - New York: Academic Press, 1976.

[5] Garey M. R., Johnson D. S. Computers and Intractability: A Guide to the Theory of NP-completeness. - New York: W. H. Freeman, 1979.

[6] Jackson M., McKenzie R. Interpreting graph colorability in finite semigroups // Int. J. Algebra and Computation. - 2006. - Vol. 16, no. 1. - P. 119-140.

[7] Hall T.E., Kublanovskii S. I., Margolis S., Sapir M. V., Trotter P. G. Algorithmic problems for finite groups and finite 0-simple semigroups // J. Pure Appl. Algebra. - 1997. - Vol. 119, no. 1. - P. 75-96.

[8] Houghton C. H. Completely 0-simple semigroups and their associated graphs and groups // Semigroup Forum. - 1977. - Vol. 14, no. 1. - P. 41-67.

[9] Kalicki J. On comparison of finite algebras // Proc. Amer. Math. Soc. - 1952.Vol. 3, no. 1. - P. 36-40.

[10] Kharlampovich O.G., Sapir M.V. Algorithmic problems in varieties // Int. J. Algebra and Computation. - 1995. - Vol. 5, no. 4-5. - P. 379-602.

[11] Kleiman Ju. G. On a basis of the product of varieties of groups // Izv. Akad. Nauk SSSR. Ser. Mat. - 1973. - Vol. 37, no. 1. - P. 95-97 [Russian; Engl. translation Math. USSR-Izv. - 1973. - Vol. 7, no. 1. - P. 91-94].

[12] Klíma O. Complexity issues of checking identities in finite monoids // Semigroup Forum. - 2009. - Vol. 79, no. 3. - P. 435-444.

[13] Kozik M. On some complexity problems in finite algebras. - PhD Dissertation. Nashville: Vanderbilt University, 2004.

[14] Kozik M. Computationally and algebraically complex finite algebra membership problems // Int. J. Algebra and Computation. - 2007. - Vol. 17, no. 8. - P. 16351666.

[15] Kozik M. A 2EXPTIME complete varietal membership problem // SIAM J. Comput. - 2009. - Vol. 38, no. 6. - P. 2443-2467.

[16] Lee E. W.H. Identity bases for some non-exact varieties // Semigroup Forum. 2004. - Vol. 68, no. 3. - P. 445-457.

[17] Lee E. W.H., Volkov M. V. On the structure of the lattice of combinatorial ReesSushkevich varieties // Proc. Int. Conf. "Semigroups and Formal Languages" in honour of the 65th birthday of Donald B. McAlister. - New Jersey: World Scientific, 2007. - P. 164-187.

[18] Lee E. W. H., Volkov M. V. Limit varieties generated by completely 0-simple semigroups // Int. J. Algebra and Computation, accepted.

[19] Mashevitsky G. I. On identities in varieties of completely simple semigroups over abelian groups // Sovremennaya Algebra [Contemporary Algebra]. - Leningrad: Leningr. State Pedagogical Inst., 1978. - P. 81-89 [Russian].

[20] Mashevitzky, G. I. An example of a finite semigroup without irreducible identity basis in the class of completely 0-simple semigroups // Uspekhi Mat. Nauk. 1983. - Vol. 38, no. 2. - P. 211-213 [Russian; Engl. translation Russ. Math. Surv. - 1983. - Vol. 38, no. 2. - P. 192-193]. 
[21] Mashevitsky G. I. Varieties generated by completely 0-simple semigroups // Polugruppy i ikh Homomorfizmy [Semigroups and their Homomorphisms]. - Leningrad: Russian State Pedagogical Univ., 1991. - P. 53-62 [Russian].

[22] Mashevitsky G. I. Matrix rank 1 semigroup identities // Comm. Algebra. - 1994. - Vol. 22, no. 9.- P. 3553-3562.

[23] Mashevitsky G.I. The pseudovariety generated by completely 0-simple semigroups // Semigroup Forum. - 1997. - Vol. 54, no. 1. - P. 83-91.

[24] Oates S., Powell M. B. Identical relations in finite groups // J. Algebra. - 1964. Vol. 1, no. 1. - P. 11-39.

[25] Ore O. Theory of Graphs. - Providence, RI: Amer. Math. Soc., 1962.

[26] Papadimitriou C. H. Computational Complexity. - Reading: Addison-Wesley Publishing Company, 1994.

[27] Perkins P. Bases for equational theories of semigroups // J. Algebra. - 1969. Vol. 11, no. 2. - P. 298-314.

[28] Pollák Gy. Arithmetics in free semigroups // Acta Sci. Math. Szeged. - 2002. Vol. 68, no. 1-2. - P. 107-115.

[29] Sapir M. V. On Cross semigroup varieties and related questions // Semigroup Forum. - 1991. - Vol. 42, no. 1. - P. 345-364.

[30] Seif S. The Perkins semigroup has co-NP-complete term-equivalence problem // Int. J. Algebra and Computation. - 2005. Vol. 15, no. 2. - P. 317-326.

[31] Shevrin L. N., Volkov M. V. Identities of semigroups // Izvestiya Vysshikh Uchebnykh Zavedenij. Matematika. - 1985. - no. 11. - P. 3-47 [Russian; Engl. translation Soviet Math. Izv. VUZ. - 1985. - Vol. 29, no 11. - P. 1-64].

[32] Székely Z. Computational complexity of the finite algebra membership problem for varieties // Int. J. Algebra and Computation. 2002. - Vol. 12, no. 6. -P. 811-823.

[33] Trahtman A. N. Graphs of identities of a completely 0-simple five-element semigroup // Ural Polytechnic Institute, Sverdlovsk, 1981, 6pp. [Russian]. (Deposited at VINITI [All-Union Institute for Scientific and Technical Information] on 07.12.81, Moscow, no. 5558-81.)

[34] Trahtman A. N. The finite basis question for semigroups of order less than six // Semigroup Forum. - 1983. - Vol. 27, no. 1-4. - P. 387-389.

[35] Trahtman, A. N. Finiteness of identity bases of 5-element semigroups // Polugruppy i ikh Homomorfizmy [Semigroups and their Homomorphisms]. - Leningrad: Russian State Pedagogical Univ., 1991. - P. 76-97 [Russian].

[36] Trahtman A. N. Identities of a five-element 0-simple semigroup // Semigroup Forum. - 1994. - Vol. 48, no. 3. - P. 385-387.

[37] Volkov M. V. On the finite basis property for semigroup varieties // Mat. Zametki. - 1989. - Vol. 45, no. 3. - P. 12-23 [Russian; Engl. translation Math. Notes. - 1989. - Vol. 45, no. 3. - P. 187-194].

[38] Volkov M. V. The finite basis problem for finite semigroups // Sci. Math. Japon. - 2001. - Vol. 53, no. 1. - P. 171-199. 